\title{
Energy expenditure and physical activity of malnourished Gambian infants
}

By L. Vasquez-Velasouez (UNU Research Fellow), Dunn Clinical Nutrition Centre, 100 Tennis Court Road, Cambridge CB2 1QL and Instituto de Nutrición de Centro America y Panama (INCAP), PO Box 11-88, Guatemala

The total energy requirements of an infant can be divided into three major components: (a) the energy expended in maintaining essential body functions (basal metabolic rate; BMR) which includes the synthetic costs of growth, (b) energy used in physical activity and thermogenesis, (c) energy deposited as new tissue.

In the past, attempts to quantify these different compartments have had variable degrees of success. For many decades, it has been possible to measure basal metabolism using classical direct and indirect calorimetry and the precision of the results depends more on the experimenter's ability to impose uniform physiological conditions than on any technical limitations. However, the measurement of total energy expenditure (TEE), and hence the energy cost of activity and thermogenesis, has so far been impossible.

The recent extension of the doubly-labelled-water (DLW) method into the field of human nutrition now makes such measurements theoretically achievable (Schoeller \& Webb, 1984; Coward et al. 1985, 1986). By combining the new technique with classical measurements of BMR it is also possible to derive estimates for the other components of expenditure.

The present paper will present preliminary findings from a study of energy expenditure in undernourished Gambian children. The results illustrate the great potential of the DLW method in field studies of this type.

\section{Material and methods}

Subjects. Results are drawn from a large study of energy expenditure in infants from birth to 3 years of age performed in the rural village of Keneba, The Gambia. A mixed longitudinal and cross-sectional design was used to assess the effect of age, malnutrition, infection and seasonality on the different components of energy expenditure. Results are presented for twenty-two infants (thirteen male and nine female), each measured at 6-monthly intervals on three different occasions, giving an age span from 2 to 29 months (mean 14 months). When completed the study will provide values from 300 measurements in The Gambia together with values from randomly-selected well-nourished British controls.

The DLW technique. Fig. 1 illustrates the principle behind the DLW method. An accurately-weighed oral dose of water labelled with the stable isotopes ${ }^{2} \mathrm{H}$ and ${ }^{18} \mathrm{O}$ is administered to the infant. The ${ }^{2} \mathrm{H}$ labels the body's water pool and ${ }^{18} \mathrm{O}$ labels both the water and bicarbonate pools which are in rapid equilibrium through the carbonic anhydrase ( $E C 4.2 .1 .1)$ reaction. The turnover rates of the two isotopes are measured by analysis of serial saliva (or urine) samples collected over a number of days. The ${ }^{2} \mathbf{H}$ rate-constant represents water turnover and the ${ }^{18} \mathrm{O}$ rate-constant represents water-pluscarbon dioxide turnover from which $\mathrm{CO}_{2}$ production can be derived by difference. Energy expenditure is calculated from $\mathrm{CO}_{2}$ production using classical indirect calorimetric calculations which require an estimate of the mean respiratory quotient (RQ) over the entire measurement period.

In the present study the isotope dose levels were $0.28 \mathrm{~g}^{18} \mathrm{O}$ and $0.10 \mathrm{~g}{ }^{2} \mathrm{H} / \mathrm{kg}$ body-weight administered using a preweighed $30 \mathrm{ml}$ syringe. Saliva samples were 


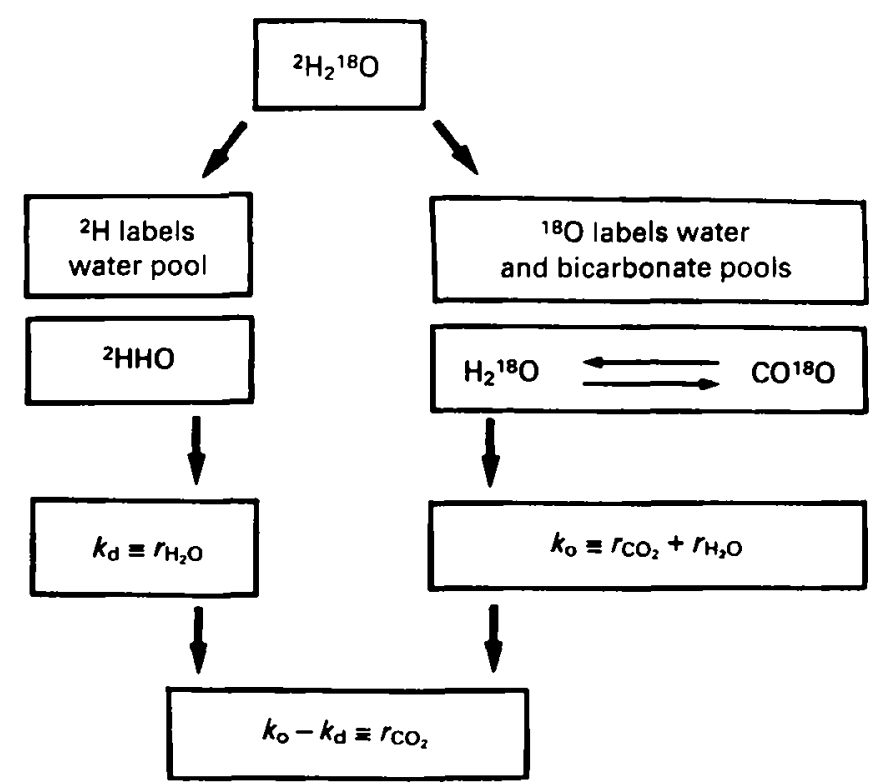

Fig. 1. A schematic explanation of the doubly-labelled-water method, where $k$ is a rate-constant for isotope disappearance, $r$ is production rate, and subscripts $d$ and $o$ refer to ${ }^{2} \mathrm{H}$ and ${ }^{18} \mathrm{O}$ respectively.

collected using dry cotton swabs and immediately transferred to sealed tubes at the following times: predose, 3, 4 and $5 \mathrm{~h}$ post-dose, and daily thereafter for $10 \mathrm{~d}$. The $10-\mathrm{d}$ measurement period spanned close to three biological half-lives of each isotope in most of the infants due to the high water turnover rates in The Gambia. Samples were stored frozen and transported to Cambridge for isotopic analysis using a VG Isogas, Aqua Syra isotope ratio mass spectrometer. Results were calculated using the Cambridge multipoint method which applies the ${ }^{2} \mathrm{H}$ and ${ }^{18} \mathrm{O}$ rate-constants to their respective dilution spaces (Coward et al. 1985, 1986).

$R Q$ and fractionation. Two assumptions are necessary in the calculations of TEE with the DLW method. First, the method needs an estimated mean RQ value in order to assign the correct energy equivalence to the $\mathrm{CO}_{2}$ produced (Black et al. 1986; Coward et al. 1986). A value of 0.85 has been used in the present paper since accurate food quotients are not yet available. It can be calculated that this may lead to a maximum underestimate of $2 \%$ of TEE in the youngest infants and a maximum overestimate of $4-5 \%$ in the older infants.

The second assumption is related to the proportion of water which is fractionated during evaporation across epithelial membranes (Coward et al. 1986; Schoeller et al. 1986). Measurements of total water turnover and insensible water losses in the Gambian infants indicate that the fractionated proportion will be close to $0 \cdot 15$. Fig. 2 shows that even if this estimate is in error by $50 \%$ this would only produce a $3 \%$ error in the estimate of TEE.

Energy available for physical activity. The energy value for physical activity and thermogenesis was obtained by subtracting from the TEE an estimated value for energy available for maintenance. Energy for maintenance was calculated using predictive equations of sleeping metabolic rate (SMR) produced from a large number of measurements carried out on Gambian children of the same age. In the present paper this compartment will be referred to simply as energy for activity since it is the most important component. 


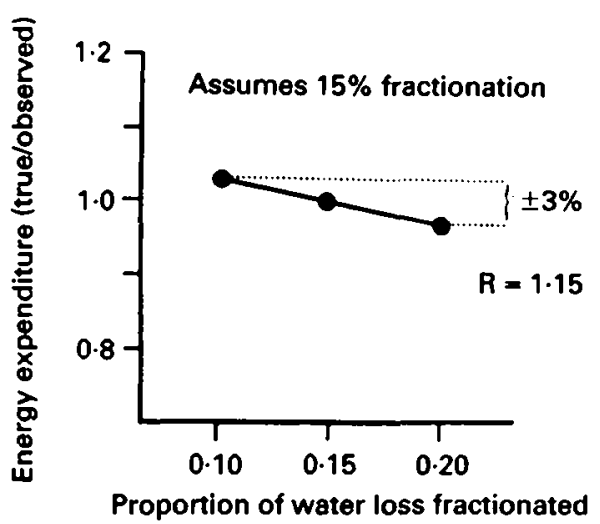

Fig. 2. The effect of variation in the proportion of water loss fractionated on total energy expenditure.

$$
\mathrm{R}=k_{\mathrm{o}} V_{\mathrm{o}} / k_{\mathrm{d}} V_{\mathrm{d}} \text {, }
$$

where $\mathrm{R}$ is ratio, $k$ is a rate-constant for isotope disappearance, $V$ is isotope dilution space and subscripts o and $\mathrm{d}$ refer to ${ }^{18} \mathrm{O}$ and ${ }^{2} \mathrm{H}$ respectively. Error is expected when the proportion of total water loss fractionated is not known.

\section{Table 1. Nutritional status of Gambian infants using National Committee for Health Statistics (1976) standards.}

(a) Percentage adequacy of weight-for-age

$\begin{array}{lrc} & n & \% \text { Total } \\ 90 \text { or }> & 22 & 33 \\ 75-90 & 37 & 56 \\ 60-75 & 7 & 11 \\ 60 \text { or }< & 0 & 0\end{array}$

(b) Percentage adequacy of weight-for-height

$\begin{array}{lrc} & n & \% \text { Total } \\ 100 \text { or }> & 18 & 27 \\ 90-100 & 24 & 36 \\ 80-90 & 24 & 36 \\ 80 \text { or }< & 0 & 0\end{array}$

\section{Results}

Nutritional status. Table 1 shows that at the time of measurement $67 \%$ of the sample were less than $90 \%$ weight-for-age using the National Committee for Health Statistics (1976) standards. The infants were also stunted, indicating chronic malnutrition, since only $36 \%$ of the sample were less than $90 \%$ weight-for-height.

TEE. Fig. 3(a) shows isotopically-measured TEE expressed as means and standard deviations at different ages. TEE increased steadily from $1883 \mathrm{~kJ}(450 \mathrm{kcal}) / \mathrm{d}$ at 3 months to $4435 \mathrm{~kJ}(1060 \mathrm{kcal}) / \mathrm{d}$ at 27 months. The coefficient of variation for individual values about the mean remained quite constant at about $19 \%$ over the age-range studied.

When expressed on a per $\mathrm{kg}$ body-weight basis (Fig. 3(b)) the TEE averaged 343 (sD 58) $\mathrm{kJ}(82$ (sD 14) $\mathrm{kcal}$ )/kg per $\mathrm{d}$ and showed no appreciable age trend. The coefficient of variation was slightly reduced to about $17 \%$. The range of values observed was $213-473$ $\mathrm{kJ}(51-113 \mathrm{kcal}) / \mathrm{kg}$ per d. 

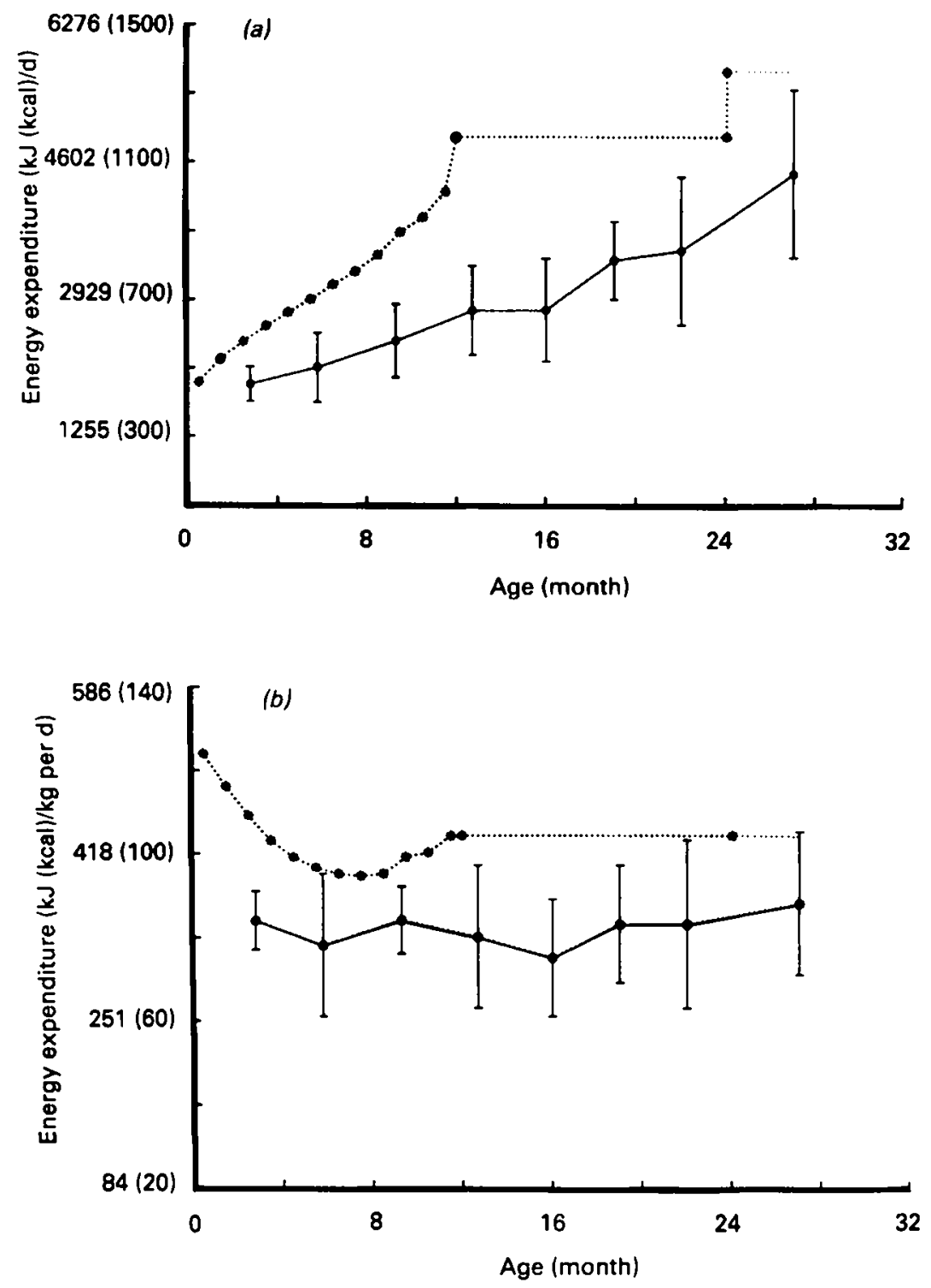

Fig. 3. Isotopic measurements of total energy expenditure ((a) kJ/d, (b) $\mathrm{kJ} / \mathrm{kg}$ per d) in Gambian infants ( () $v$. age. Values are means and 1 SD represented by vertical bars. (*....*), Food and Agriculture Organization/ World Health Organization/United Nations University (1985) recommended requirements.

The current Food and Agriculture Organization/World Health Organization/United Nations University (FAO/WHO/UNU) (1985) estimates of the energy requirements of healthy infants are shown in Fig. 3(a,b). The Gambian measurements of TEE follow the same trend as the recommended intakes, but are considerably lower at all ages. Part of this difference can be accounted for by the fact that the FAO/WHO/UNU (1985) values include an allowance for growth which is not contained in the estimates of TEE. However, even allowing for the energy stored as new tissue, a substantial difference remains. 


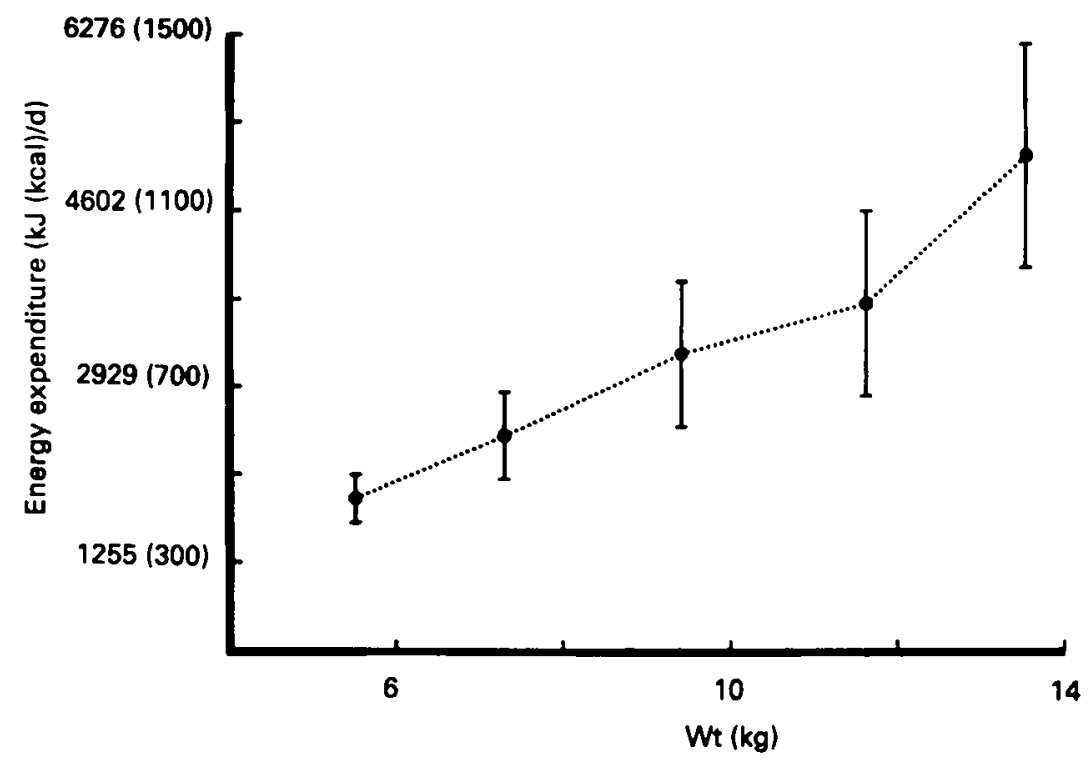

Fig. 4. Isotopic measurements of total energy expenditure in Gambian infants $v$. body-weight.

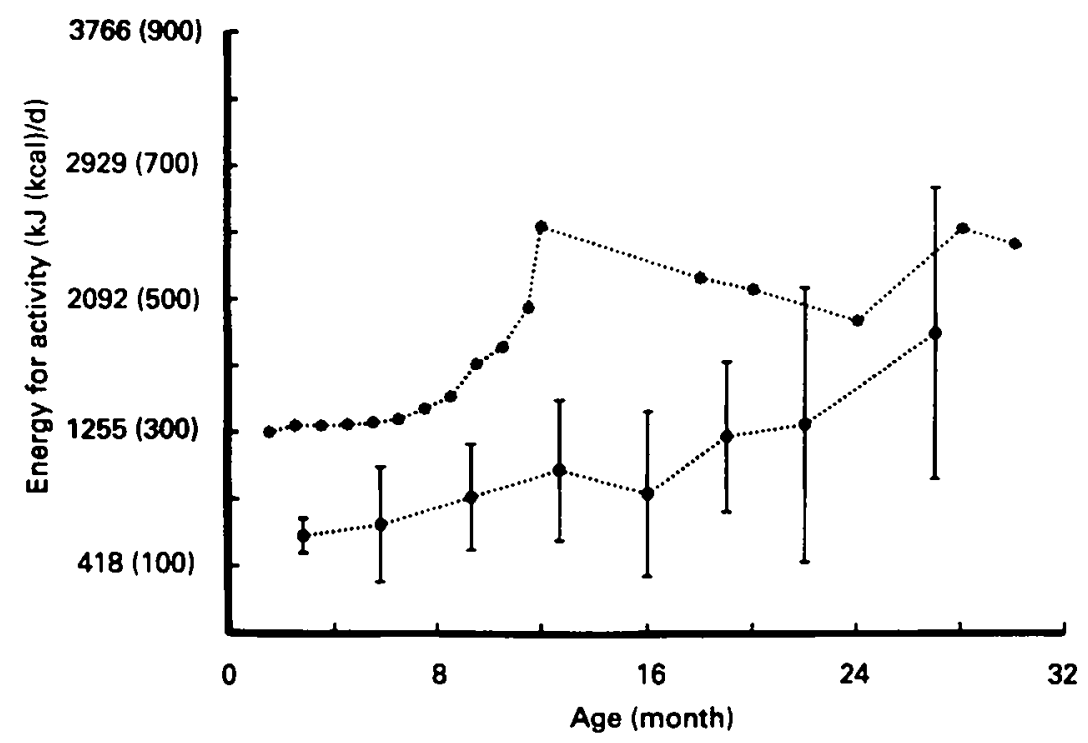

Fig. 5. Energy available for physical activity (total energy expenditure minus predicted basal metabolic rate) in Gambian children. Values are means and 1 sD represented by vertical bars. (*...*), Food and Agricultural Organization/World Health Organization/United Nations University (1985) recommended requirements.

Correlation analysis using age, height and weight as independent variables demonstrated that body-weight was the primary determinant of TEE and that the relationship was essentially linear (Fig. 4). Although this correlation appears close when viewed on 
this scale it should be noted that there is a twofold range in TEE at any given weight. A $19 \%$ coefficient of variation was found.

Energy available for physical activity. Fig. 5 shows the estimated amount of energy expended on physical activity and thermogenesis derived as TEE-BMR. There was a steady increase in this component from about $628 \mathrm{~kJ}(150 \mathrm{kcal}) / \mathrm{d}$ at 3 months to about $1883 \mathrm{~kJ}(450 \mathrm{kcal}) / \mathrm{d}$ at 27 months. The implied allocation for activity and thermogenesis in the FAO/WHO/UNU (1985) recommendations was derived by subtracting predicted BMR from the total recommended intake. Once again the FAO/WHO/UNU values still contain a small additional component for energy stored during growth, but this accounts for only a fraction of the difference between the observed and recommended values.

\section{Discussion}

The non-intrusiveness and ease of application of the DLW method could make it ideally suited to field studies involving young children in whom alternative methods such as indirect calorimetry can be used only during sleep. However, it is impossible to make any direct assessments of the accuracy and precision of the new method in this age group. The ideal validation protocols involve long-term simultaneous comparisons of $\mathrm{CO}_{2}$ production measured by whole-body indirect calorimetry and by DLW. These have been performed in adults (Schoeller \& Webb, 1984; Coward et al. 1985) and in premature infants (Roberts et al. 1986). In general the results confirm the conclusion from the literature relating to animals that the method works well (Schoeller, 1984) and in the absence of any known confounding factors, it seems reasonable to interpolate from these studies to conclude that DLW works in principle for infants. The other estimates of errors arising from the $\mathrm{RQ}$ and fractionation assumptions can be computed from first principles in the same way as for adults and are generally very small (Black et al. 1986; Coward et al. 1986).

The present study has confirmed that the method is well-tolerated by infants and straightforward to apply even under difficult field conditions. The preliminary findings presented here form part of a larger study and should not be considered definitive, particularly as they relate to just twenty-two infants measured repeatedly in a mixedlongitudinal manner. The completed study will provide information on both intra- and inter-individual differences in energy expenditure and on influencing factors, such as age, sex, size, body composition, acute and chronic malnutrition, infection and seasonality. However, a number of useful observations can already be made.

The variability in TEE between individuals is very large: coefficient of variation is $19 \%$ for children of the same age and weight. This supports Widdowson's (1947) conclusion based on measurements of food intake, but is perhaps even more surprising since the current estimates are of expenditure rather than intake and cover a 10-d period. Both these factors would be predicted to reduce the component of intra-individual variability which must contribute to the inter-individual observations.

The mean value of $343 \mathrm{~kJ}(82 \mathrm{kcal}) / \mathrm{kg}$ per d for TEE was similar to estimates presented by Fjeld et al. (1988). The range of $213-473 \mathrm{~kJ}(51-113 \mathrm{kcal}) / \mathrm{kg}$ per d clearly includes some suspiciously low values. However, it should be noted that none of the estimates of TEE-BMR were negative even when predicted BMR values were used. In fact, correlation analysis of independent measurements of TEE and BMR made in the same children show a significant positive correlation. Thus children with a low TEE also tend to have a low BMR which leaves an intuitively reasonable amount of energy available for physical activity.

The FAO/WHO/UNU (1985) estimates of requirements have been included only to provide a perspective for the new measurements. It should be remembered that the 
estimates of requirements include an allowance for the energy deposited as new tissue (about $259 \mathrm{~kJ}(62 \mathrm{kcal}) / \mathrm{d}$ at 2 months falling to $67 \mathrm{~kJ}(16 \mathrm{kcal}) / \mathrm{d}$ at 24 months) and a $5 \%$ additional allowance to compensate for the fact that levels of physical activity may be less than optimal for good health. In spite of this, however, the current measurements do appear to be substantially lower, and it is unlikely that increasing the sample size will make much difference to the mean values. Full interpretation of these results will have to await information from the well-nourished controls.

In conclusion, the theoretical estimates of the accuracy of the DLW method indicate it to be superior to any other available method based either on measurements of intake or expenditure. The method has other advantages in terms of non-intrusiveness, absence of an observer effect and integration of expenditure over long periods. The FAO/WHO/ UNU (1985) report stated that ' . . in principle it would be desirable to determine the requirements of children in the same way as for adults, from measurements of energy expenditure' and this now appears to be possible.

\section{REFERENCES}

Black, A. E., Prentice, A. M. \& Coward, W. A. (1986). Human Nutrition: Clinical Nutrition 40C, 381-391.

Coward, W. A., Prentice, A. M., Murgatroyd, P. R., Davies, H. L., Cole, T. J., Sawyer, M., Goldberg, G. R., Halliday, D. \& Macnamara, J. P. (1985). In Human Energy Metabolism: Physical Activity and Energy Expenditure Measurements in Epidemiological Research Based upon Direct and Indirect Calorimetry, Euro-Nut Report no. 5, pp. 126-128 [A. J. H. Van Es, editor]. Den Haag: CIP-gegevens koninklijke.

Coward, W. A., Roberts, S. B., Prentice, A. M. \& Lucas, A. (1986). Clinical Nutrition and Metabolic Research. Proceedings of the 7th Congress of the European Society of Parenteral-Enteral Nutrition (ESPEN). Munich 1985, pp. 169-177 [G. Dietze, A. Grumert, G. Kleinberger, G. Wolfram, editors]. Basel: Karger.

Fjeld, C. R., Schoeller, D. A. \& Brown, K. H. (1988). Proceedings of the Nutrition Society 47, 227-231.

Food and Agriculture Organization/World Health Organization/United Nations University (1985). Energy and Protein Requirements. Technical Report Series no. 724. Geneva: WHO.

National Committee for Health Statistics (1976). Growth Charts HRA 76-1120, 25, 3. Rockville, Md: United States Department of Health, Education and Welfare, Public Health Service, Health Resources Administration.

Roberts, S. B., Coward, W. A., Schlingenseipen, K.-H., Nohria, V. \& Lucas, A. (1986). American Journal of Clinical Nutrition 44, 315-322.

Schoeller, D. A. (1984). Human Nutrition: Clinical Nutrition 38C, 477-480.

Schoeller, D. A., Leitch, C. A. \& Brown, C. (1986). American Journal of Physiology 251, R1137-R1143.

Schoeller, D. A. \& Webb, P. (1984). American Journal of Clinical Nutrition 40, 153-158.

Widdowson, E. M. (1947). A Study of Individual Children's Diets, MRC Special Report Series no. 257. London: H.M. Stationery Office. 